\title{
The Galactic Center Source IRS 13E: a Star Cluster
}

\author{
Jean-Pierre Maillard ${ }^{1}$, Thibaut Paumard ${ }^{1}$, Susan Stolovy $^{2}$, and François Rigaut ${ }^{3}$ \\ ${ }^{1}$ Institut d'Astrophysique de Paris (CNRS), 98b Blvd Arago, 75014, Paris, FRANCE \\ 2 SIRTF Science Center, CalTech, MS 220-6, Pasadena, CA 91125, USA \\ ${ }^{3}$ Gemini North Headquarter, Hilo, HI 96720, USA
}

Key words Galactic Center, star cluster, WR star, infrared, adaptive optics

\begin{abstract}
High spatial resolution, near-infrared observations of the Galactic Center source, close to Sgr A*, known historically as IRS13, are presented. These observations include ground-based adaptive optics images in the H, K' and L bands, HST-NICMOS observations in filters between 1.1 and $2.2 \mu \mathrm{m}$, and spectroimaging data in the $\mathrm{He} \mathrm{I} 2.06 \mu \mathrm{m}$ line and the $\mathrm{Br} \gamma$ line. Analysis of all these data has made possible the resolution of the main component, IRS 13E, into a cluster of seven individual stars within a projected diameter of $\sim 0.5^{\prime \prime}(0.02 \mathrm{pc})$, and to build their SED. The main sources, 13E1, 13E2, 13E3 (a binary), and 13E4, are hot stars of different nature. 13E2 and 13E4 are emission line stars. The spectral type of the various members goes from O5I to WR, including dusty WRs like IRS 21 (Tanner et al. 2002). All these sources have a common westward proper motion. Two weaker sources, 13E5 and 13E6, are also detected within the compact cluster, with 13E5 proposed as another dusty WR and 13E6 as a O5V star. An extended halo seen around the cluster, part of the mini-spiral of dust is particularly enhanced in the L band. It is interpreted as a contribution of the scattered light from the inner cluster and the thermal emission from the dust. IRS 13E is proposed to be the remaining core of a massive, young star cluster which was disrupted in the vicinity of $\mathrm{Sgr} \mathrm{A}^{\star}$, and hence, the possible source of the young stars in the central parsec, from the helium stars to the S stars.
\end{abstract}

\section{Introduction}

In the early mapping works of the central parsecs, a spot named IRS 13, bright at all near-infrared wavelengths, was reported, located approximately $3.6^{\prime \prime}$ south-west of Sgr A ${ }^{\star}$. It was later resolved into two sources in the K band separated by $\sim 1.2^{\prime \prime}$, IRS 13E and IRS 13W (Simon et al. 1990). From spectroscopic studies in the same band, IRS 13W was identified as a cool star (Krabbe et al. 1995) and IRS 13E as an emission line source with strong $\mathrm{HeI} 2.058,2.112 \mu \mathrm{m}, \mathrm{Br} \gamma$ line and other Brackett lines up to $\mathrm{Br} 12$ (Genzel et al. 1996), typical of the helium stars present in the central parsec. The first adaptive optics (AO) map of IRS 13E in the K band obtained on the CFH Telescope was published by Paumard et al. (2001), showing that the source resolved into two equally bright components 13E1 and 13E2, plus a third weaker component called 13E3. Since the spectra of IRS 13E does not have the same spatial resolution as the images, the identification of the associated spectral type is subject to caution. In the centimetric domain, Zhao and Goss (1998) found IRS 13 as the brightest radio continuum source after Sgr A ${ }^{\star}$ at the Galactic Center. The detection of a discrete X-ray source from CHANDRA at the position of IRS 13 (Baganoff $e t$ al. 2001) was another element making IRS 13 a source of special interest. The high resolution images of the central parsec currently obtained at various wavelengths in the infrared provide the possibility of studying this peculiar Galactic Center source in detail. A complete description of the present work can be found in the companion paper of Maillard et al. (2003). 


\section{New data on IRS 13}

Calibrated ground-based AO data from several telescopes and space-based NICMOS data in the near infrared, all containing IRS 13 in their field, plus some spectroscopic data, and the proper motions of the sources of the IRS 13 field, have all been combined.

\subsection{High-angular resolution data}

The AO data come from two different systems, Gemini North (Graves et al. 1998) for the $\mathrm{H}$ and the Kp $(2.12 \mu \mathrm{m}, \mathrm{FWHM} 0.41 \mu \mathrm{m})$ bands, and ESO 3.6-m telescope (Clénet et al. 2001) for the L band. Medium (M) and wide-band (W) filters, respectively centered at 1.1, 1.45, 1.60, 2.22 $\mu \mathrm{m}$ (coded F110M, F145M, F160W and F222M) and two close narrow-band (N) filters (F187N centered on the $1.87 \mu \mathrm{m}$ Pa $\alpha$ line and F190N) were used in observing the stars at the inner parsec of the Galactic Center with the NICMOS cameras on board HST.

A small $\simeq 2.5^{\prime \prime} \times 2.5^{\prime \prime}$ region of the image, roughly centered on IRS $13 \mathrm{E}$, was analyzed for each filter. All these high-resolution, multi-band images have provided spectrophotometric information on the IRS 13 sources and its environment, from 1 to $4 \mu \mathrm{m}$. The Gemini AO data were calibrated by linear interpolation based on one bright, hot star of the field under study, from the calibrated NICMOS data, between the F160W and F190N photometry for the H band, F190N and F222M for the Kp band. The star detection and photometry was made with the StarFinder procedure (Diolaiti et al. 2000) for all the images. For the AO images a deconvolution code called MCS (Magain et al. 1998) was applied. For the $H$ and the Kp images the width of the synthetic PSF was equal to $0.040^{\prime \prime}$ and to $0.192^{\prime \prime}$ for the L-band image, i.e. a gain in resolution respectively of a factor 4.5 in $\mathrm{H}, 4.3$ in $\mathrm{Kp}$ and 1.5 in L.

\subsection{Spectroscopic data}

The only high-spatial resolution images giving spectroscopic information are contained in the NICMOS narrow-band images from the F187N filter. By subtraction of F190N, a narrow-band filter in the nearby continuum, from F187N, a map of the $1.87 \mu \mathrm{m} \mathrm{Pa} \alpha$ emission was obtained (Stolovy et al. 1999). This map shows the distribution of the ionized gas and stellar spots from Pa $\alpha$ emission in the atmosphere of the hot stars. The Br $\gamma$ and $2.06 \mu \mathrm{m}$ He I line profiles at IRS 13E from BEAR spectro-imagery, an imaging FTS (Maillard 2000), were used as complementary information to help define the spectral type of the underlying stars. The IRS 13 complex is located in a region of intense interstellar emission. The data cube was particularly useful to correct the two emission line profiles from the interstellar emission, leaving fully resolved stellar profiles respectively at 21.3 and $52 \mathrm{~km} \mathrm{~s}^{-1}$ resolution.

\subsection{Proper motions}

The proper motions of the IRS 13 sources and the sources contained in the surrounding $2.5^{\prime \prime} \times 2.5^{\prime \prime}$ were obtained from Ott et al. (2003) who conducted an analysis of ten years of SHARP data (Eckart et al. 1995), providing more than 1000 proper motions in the central parsec.

\section{Results}

From the deconvolution analysis of the $2.5^{\prime \prime} \times 2.5^{\prime \prime}$ field including IRS 13, 20 individual sources were identified. IRS $13 \mathrm{E}$ is decomposed into seven sources, respectively named 13E1, 13E2, 13E3A and B, $13 \mathrm{E} 4,13 \mathrm{E} 5$ and 13E6. The name 13E3A and B is proposed for the two components of the source 13E3 which appears double only after deconvolution, in the $\mathrm{H}$ and $\mathrm{Kp}$ bands. The positions of all the sources and their proper motions, estimated for most of them, are given in Fig. 1. Their observed photometry in the H, $\mathrm{K}$ and $\mathrm{L}$ bands is presented in Table 1. In the F187N - F190N image only 13E2 and 13E4 are remaining, indicative that these two sources are emission stars. 


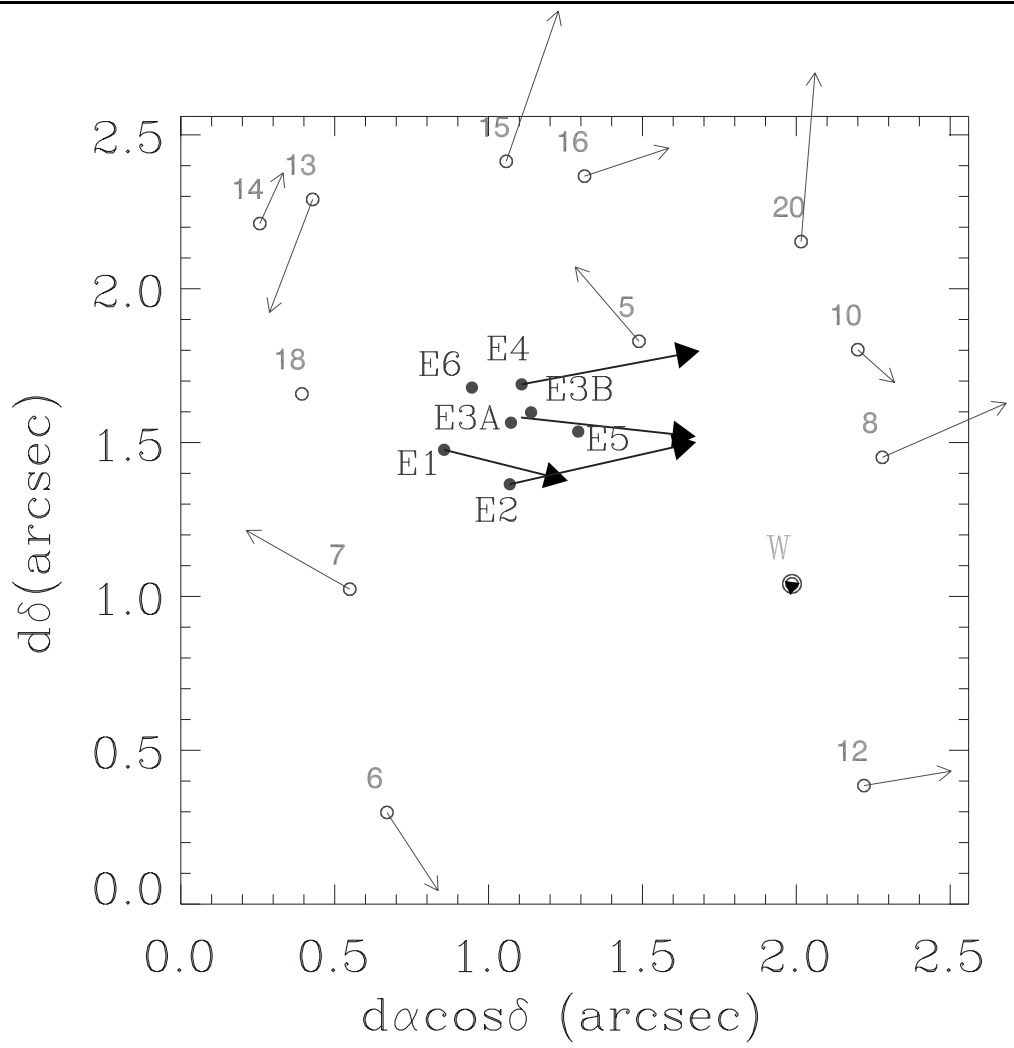

Fig. 1 The IRS 13 field with the star identification. The vector associated with most of the stars represents the amplitude in velocity and the direction of proper motion measured from SHARP data by Ott et al. (2003). For E3A and $\mathrm{E} 3 \mathrm{~B}$ only the barycenter proper motion is determined. The origin of the field corresponds to $-2.19^{\prime \prime} \mathrm{W}$ and $-3.00^{\prime \prime} \mathrm{S}$ with respect to Sgr $\mathrm{A}^{\star}$.

From the flux measurements made in 8 bands, between 1.1 and $3.5 \mu \mathrm{m}$, it was possible to obtain dereddened spectrophometry of the IRS 13 sources. This requires the adoption of an $A_{V}$ value and an extinction law. The mean value of $A_{V}=31.1$ is known to vary strongly with location within the central parsecs (Scoville et al. 2003). The local value was derived utilizing two constraints: IRS $13 \mathrm{~W}$ is known to be a cool oxygen star, and IRS 13E2 and 13E4 are hot stars, as seen in Pa $\alpha$ imaging. A value of $A_{V}=35$ was adopted and was assumed to be valid over the small field around IRS 13. $A_{V}$ could be pretty well constrained assuming that IRS 13E2 is a WR star with $\mathrm{T}_{\text {eff }}>25,000 \mathrm{~K}$. The IRS 13E2 spectral type is based on the $\mathrm{Pa} \alpha$ imaging, but also on Fabry-Pérot spectro-imaging behind the CFHT-AO system where IRS 13E2 is detected as the only source of the broad $2.06 \mu \mathrm{m}$ He I emission line (Clénet et al. 2003). With the spectral range under study, from about 1 to $4 \mu \mathrm{m}$, beyond a temperature of $25,000 \mathrm{~K}$ we are in the Rayleigh-Jeans regime, and the shape of the SED becomes constant in a $\log F(\lambda)$ versus $\lambda$ diagram. $A_{V}$ can be adjusted to bring the data points parallel to the SED. However, the fit of the dereddened data has to be made as the sum of two black-body curves since most of the sources have an infrared excess, a signature of thermal dust emission. The adjustments is made with four parameters for each IRS 13 star, by Coe $f_{1} \times B B\left(T_{1}\right)+$ Coe $_{2} \times B B\left(T_{2}\right)$. The $\mathrm{T}_{1}$ temperature being the high-temperature component, is mainly determined by the data points between 1 and $2.5 \mu \mathrm{m}$, and $\mathrm{T}_{2}$ by the 2 to $4 \mu \mathrm{m}$ points. If for the fitting, $\mathrm{T}_{1}$ becomes $\geq 25,000 \mathrm{~K}$ the temperature is fixed at $25,000 \mathrm{~K}$. The four final parameters are presented in Table 2. The dereddened points and the fits are shown on Fig. 2. Several stars are very hot stars, i.e. with 
a $\mathrm{T}_{\text {eff }} \geq 25,000 \mathrm{~K}$. The NICMOS F110M and the $\mathrm{L}$ data are essential to provide the maximum constraints for the SED of each source.

\section{Nature of the IRS 13E sources}

From the main results reported in the previous section an identification of the spectral type of the seven components of IRS 13E can be derived.

Table $1 \mathrm{H}, \mathrm{K}$ and L photometry of the IRS 13E cluster and the nearby field stars

\begin{tabular}{|l|ccc|c|c|}
\hline ID & $H$ & $K$ & $L$ & $H-K$ & $K-L$ \\
\hline \hline W & 14.51 & 11.22 & 8.92 & 3.29 & 2.29 \\
E1 & 12.71 & 10.90 & 8.59 & 1.81 & 2.31 \\
E2 & 13.02 & 10.95 & 7.73 & 2.07 & 3.21 \\
E4 & 14.34 & 11.64 & & 2.70 & \\
5 & 14.28 & 11.82 & & 2.47 & \\
6 & 14.68 & 12.05 & & 2.63 & \\
7 & 14.38 & 12.10 & & 2.28 & \\
8 & 14.82 & 12.14 & & 2.68 & \\
E3A & 16.44 & 12.38 & 7.50 & 4.06 & 4.89 \\
10 & 15.51 & 12.71 & & 2.80 & \\
E3B & 16.93 & 12.98 & 7.92 & 3.95 & 5.06 \\
12 & 15.89 & 13.19 & & 2.69 & \\
13 & 15.91 & 13.26 & & 2.65 & \\
14 & 15.91 & 13.33 & & 2.58 & \\
15 & 16.61 & 14.12 & & 2.49 & \\
16 & 16.62 & 14.13 & & 2.49 & \\
E5 & 18.15 & 14.20 & 8.48 & 3.95 & 5.71 \\
18 & 16.77 & 14.43 & & 2.34 & \\
E6 & 16.20 & 14.48 & & 1.73 & \\
20 & 17.26 & 14.70 & & 2.56 & \\
\hline
\end{tabular}

Table 2 Fitting parameters of the SED of the IRS 13E sources and IRS 13W The spectral type of each source as discussed in Sect. 5 is summarized in the last column.

\begin{tabular}{|l|rrrr|c|}
\hline Star & Coef $_{1}$ & T $_{1} \mathrm{~K}$ & Coef $_{2}$ & T $_{2} \mathrm{~K}$ & Sp. Type \\
\hline W & 23.00 & $\mathbf{2 6 0 0}$ & 1700 & $\mathbf{6 5 0}$ & M5III \\
E1 & 0.500 & $\geq \mathbf{2 5 0 0 0}$ & 12000 & $\mathbf{5 5 0}$ & O5I \\
E2 & 0.450 & $\geq \mathbf{2 5 0 0 0}$ & 40000 & $\mathbf{5 5 0}$ & WC9 \\
E4 & 0.070 & $\geq \mathbf{2 5 0 0 0}$ & 45 & $\mathbf{1 5 5 0}$ & O5IIIe \\
E3A & 0.460 & $\mathbf{3 8 0 0}$ & 33000 & $\mathbf{6 1 0}$ & d. WR \\
E3B & 0.375 & $\mathbf{3 8 0 0}$ & 29000 & $\mathbf{5 8 0}$ & d. WR \\
E5 & 0.070 & $\mathbf{6 0 0 0}$ & 9800 & $\mathbf{6 3 0}$ & d. WR \\
E6 & 0.008 & $\geq \mathbf{2 5 0 0 0}$ & & & O5V \\
\hline
\end{tabular}

${ }^{a}$ dusty Wolf-Rayet star 


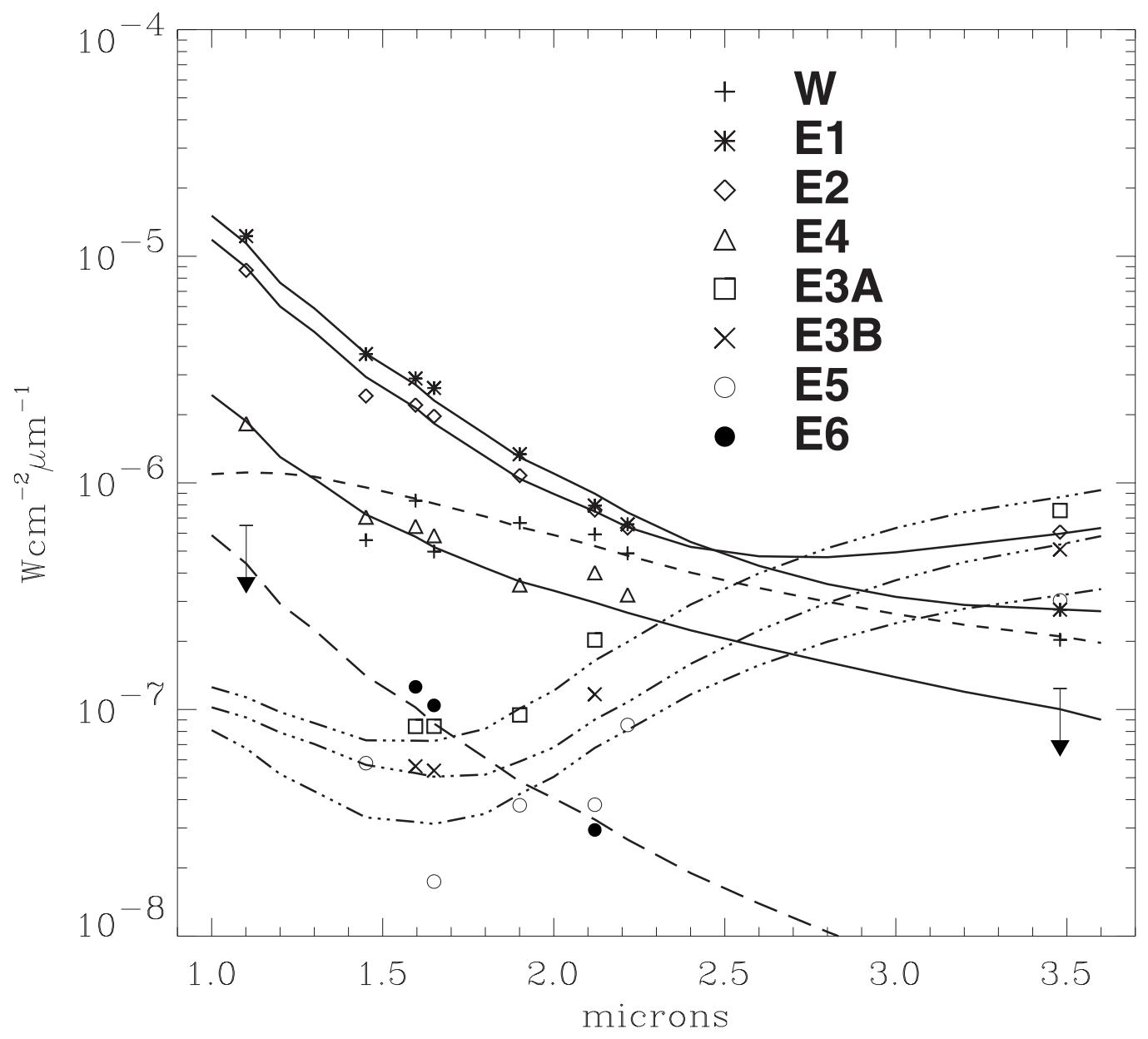

Fig. 2 Dereddened flux in $\mathrm{W} \mathrm{cm}^{-2} \mu \mathrm{m}^{-1}$ for $A_{V}=35$. The top of the arrows represents the upper limit of the detectable flux in the $1.1 \mu \mathrm{m}$ and the L-band filters. The various lines represent the best fitting between 1 and $4 \mu \mathrm{m}$ of the data points from a two-component model with the parameters of Table 2.

\subsection{E1, 13E2 and 13E4}

The source 13E1 is a bright, blue star, but with no detected emission at Pa $\alpha$. From its luminosity and its $T_{\text {eff }}$, IRS 13E1 is proposed to be close to a O5I main sequence star. 13E2 and 13E4 are two emission line stars, 13E2 being brighter in $\mathrm{Pa} \alpha$ by a factor 2. From the BEAR data, the $2.06 \mu \mathrm{m}$ He I line is a broad line $\left(\sim 900 \mathrm{~km} \mathrm{~s}^{-1} \mathrm{FWHM}\right)$ while the $\mathrm{Br} \gamma$ line is narrow ( $\left.\sim 215 \mathrm{~km} \mathrm{~s}^{-1} \mathrm{FWHM}\right) .13 \mathrm{E} 2$ is reported as the only He I emitter (Clénet et al. 2003). As a broad-line, helium-rich star, 13E2 is proposed as a Wolf-Rayet type star, from the criterion on the linewidth developed in Paumard et al. (2001). By analogy with similar stars in the central parsec the source should be more precisely a WC9 star. 13E4 is a blue star which shows a narrow emission line in $\operatorname{Pa} \alpha$ but no helium emission line. Therefore, this star is much less evolved than 13E2. It can be proposed as a O5IIIe, since it is weaker than E1 and has hydrogen lines in emission. 


\subsection{E3A, 13E3B and 13E5}

On Fig. 2 these three stars (dash-dotted lines) have a similar SED. They are adjusted by a strong, cool component at $\simeq 600 \mathrm{~K}\left(\right.$ Coef $_{2}$ is high) and a weak, hotter component of a few thousands K. This adjustment can be compared to the fitting of the SED of IRS 21 (Tanner et al. 2002) fitted by a two-component model, the near-infrared scattered light from the central source peaking at $\simeq 3.8 \mu \mathrm{m}(760 \mathrm{~K})$, and the mid-infrared re-emitted light from a dust shell at $\sim 250 \mathrm{~K}$. Tanner et al. (2003) conclude that this source is a dusty WR star, experiencing rapid mass loss, and the other red, featureless spectrum sources along the Northern Arm IRS $1 \mathrm{~W}, 2,3,5$, and 10W, as well. By analogy, we conclude that the three red sources within the IRS 13E complex, also located in the dusty part of the mini-spiral are dusty WR stars.

\subsection{E6}

The source IRS 13E6 is another blue star, much weaker than 13E1, 13E2 and 13E4, with $\mathrm{K}_{\text {mag }}=14.5$ (Table 1). The image in the $\mathrm{L}$ band is not deep enough to detect it at this wavelength to confirm that this star is also embedded in the same concentration of dust than the other IRS 13E sources. From its color and magnitude IRS 13E6 can be considered as close to a O5V type star. Without further indication, only from the fact that all the other stars in IRS 13E are hot stars, we assume that IRS 13E6 belongs also to the same complex.

\section{Model of IRS 13E as the remaining core of a massive star cluster}

IRS $13 \mathrm{E}$ appears as only composed of hot, massive stars, with at least 7 stars within $0.5^{\prime \prime}$. The common direction and comparable amplitude of the proper motions of the main components is a decisive argument to indicate that $13 \mathrm{E} 1,13 \mathrm{E} 2,13 \mathrm{E} 3 \mathrm{~A} / \mathrm{B}$ and $13 \mathrm{E} 4$ are physically bounded. The source previously called IRS $13 \mathrm{E}$ is likely a compact star cluster. Furthermore, its composition suggests a young star cluster of a few $\times 10^{6}$ yr old, since several members are identified as having already reached the WR stage.

The presence of such a compact cluster with a limited number of members raises the question of its origin. First, it can be noticed that each component has many other examples of stars of the same spectral type in the central parsecs. However, the large abundance of massive stars, which are very rare elsewhere in the Galaxy, remains one of the major mysteries of this region of the Milky Way. Since star formation would be difficult due to the strong tidal forces from the Sgr A* black hole, Gerhard (2001) made the interesting hypothesis that the central parsec He I stars, the most prominent of the massive young stars, might be the remains of a dissolved young cluster, disrupted in the vicinity of the central black-hole. Kim et al. (2003) tested this idea for different cluster masses and different initial orbit radii. They came to the conclusion that some simulations can be regarded as possible candidates for the origin of the central parsec cluster. With its exceptional concentration of massive stars, very close to Sgr A*, all bounded together, we propose that IRS 13E might be the remaining core of such a massive cluster which was disrupted by Sgr $A^{\star}$. The analysis of the 12 other stars identified in the IRS 13 field (Fig. 1) conducted as for the IRS 13E cluster sources, made possible to separate the sources in two categories, 9 red stars $\left(\mathrm{T}_{\text {eff }}\right.$ from 2800 to $5000 \mathrm{~K}$ ) and 3 blue stars $\left(\mathrm{T}_{\text {eff }} \geq 25,000 \mathrm{~K}\right)$. The red stars are members of the most numerous population of the central parsecs, which is an old population of $\mathrm{K}, \mathrm{M}$ and AGB stars, to which belongs also IRS $13 \mathrm{~W}$. The blue stars should be members of the most recent stellar population. On the other hand, the blue stars are comparable in magnitude and color to the stars of the S-cluster (Gezari et al. (2002) detected around Sgr A*. The IRS 13E cluster itself contains also one of such lower mass blue stars (Table 2). Hence, the hot stars, including the $S$ and the helium stars, could come from the same initial massive cluster, and complete its IMF. However, more simulations are needed to validate this hypothesis.

Another aspect of IRS 13 is the detection of a discrete X-ray emission within $1^{\prime \prime}$ positional accuracy (Baganoff et al. 2001). IRS 13E as a star cluster might be the X-ray source, by the colliding winds of all the close, hot, mass-losing stars. An example of such a source can be provided by the detection of a 
discrete X-ray source at the position of the core of the Arches cluster (Yusef-Zadeh et al. 2002). A better astrometry of the X-ray source at IRS 13 could help confirm this assumption, consistent with IRS 13E as the remaining core of a massive star cluster.

\section{References}

Baganoff, F.K., Maeda, Y., Morris, M., Bautz, M.W, Brandt, W.N., Cui, W., Doty, J.P., Feigelson, E.D., Garmire, G.P., Pravdo, S.H., Ricker, G.R., \& Townsley, L.K. 2001, ApJ, astro-ph/01002151

Clénet, Y., Rouan, D., Gendron, E., Montri, J., Rigaut, F., Léna, P. \& Lacombe, F. 2001, A\&A, 376, 124

Clénet, Y., Lacombe, F., Gendron, E., \& Rouan, D. 2003, these proceedings

Diolaiti, E., Bendinelli, O., Bonaccini, D., Close, L., Currie, D. \& Parmeggiani, G. 2000, A\&AS, 147, 335

Eckart, A., Genzel, R., Hofmann, R., Sams, B.J., \& Tacconi-Garman, L.E. 1995, ApJ, 445, L23

Genzel, R., Thatte, N., Krabbe, A., Kroker, H., \& Tacconi-Garman, L.E. 1996, ApJ, 472, 153

Gezari, S., Ghez, A.M., Becklin, E.E., Larkin, J., McLean, I.S., Morris, M. 2002., ApJ, 576, 790

Gerhard, O. 2001, ApJ, 546, L39

Graves, J.E., Northcott, M.J., Roddier, F.J., Roddier, C.A., \& Close, L.M. 1998, SPIE, 3353, 34

Krabbe, A., Genzel, R., Eckart, A., Najarro, F., Lutz, D., Cameron, M., Kroker, H., Tacconi-Garman, L.E., Thatte, N., Weitzel, L., Drapatz, S., Geballe, T., Sternberg, A., \& Kudritzki, R.P. 1995, ApJ, 447, L95

Kim, S.S., Morris, M., \& Figer, D.F. 2003, these proceedings

Maillard, J.P., 2000, in Imaging the Universe in 3 Dimensions, ed. E. van Breughel \& J. Bland-Hawthorn, ASP Conf. Ser., 195, 185

Maillard, J.P., Paumard, T., Stolovy, S., \& Rigaut, F. 2003, A\&A, submitted

Magain, P., Courbin, F. \& Sohy, S. 1998, ApJ, 494, 472

Ott, T., Genzel, R., Eckart, A., \& Schödel, R. 2003, these proceedings

Paumard, T., Maillard, J.P., Morris, M., \& Rigaut, F. 2001, A\&A, 366,466

Simon, M., Chen, W.P., Forrest, W.J., Garnett, J.D., Longmore, A.J., Gauer, T., \& Dixon, R.I. 1990, ApJ, 360, 95

Scoville, N.Z., Stolovy, S.R., Rieke, M., Christopher, M., \& Yusef-Zadeh, F. 2003, these proceedings

Stolovy, S.R., McCarthy, D.W., Melia, F., Rieke, G., Rieke, M.J., \& Yusef-Zadeh, F. 1999, in The Central Parsecs of the Galaxy, ed. H. Falcke, A. Cotera, W.J. Duschl, F. Melia, M.J. Rieke, ASP Conf. Ser., 186, 39

Tanner, A., Ghez, A.M, Morris, M., Becklin, E.E., Cotera, A., Ressler, M., Werner, M., \& Wizinovitch, P. 2002, ApJ, 575, 860

Tanner, A., Ghez, A.M, Morris, M., \& Becklin, E.E. 2003, these proceedings

Yusef-Zadeh, F., Law, C., Wardle, M., Wang, Q.D., Fruscione, A., Lang, C.C., \& Cotera, A. 2002, ApJ, 570, 665

Zhao, J.H., \& Goss, W.M. 1998, ApJ, 499, L163 